\title{
Evaluation of the Utilization Patterns of Leukotriene Modifiers in a Large Managed Care Health Plan
}

PAUL G. LAKOMSKI, RPh, MBA, and MONA CHITRE, PharmD, CGP

\begin{abstract}
OBJECTIVE: To assess the utilization of leukotriene modifiers (LM) relative to national guidelines and to investigate possible emergency room utilization differences for LMs as monotherapy versus inhaled corticosteroid (ICS) monotherapy or combination ICS and LM therapy.

METHODS: The utilization of leukotriene modifiers (montelukast sodium, zafirlukast, and zileuton), concurrent inhaled steroids (beclomethasone, budesonide, flunisolide, fluticasone, and triamcinolone), beta-agonists (albuterol, bitolterol, formoterol, isoetharine, levalbuterol, metaproterenol, pirbuterol, salmeterol, and terbutaline) and low-sedating antihistamines ([LSAs] cetirizine, desloratadine, fexofenadine, and loratadine) were assessed from the drug claims database of a large health insurer for dates of service for the 12-month period from September 1, 2001, through August 31, 2002. New-start LM patients were identified as having no previous LM drug claim within a 180 -day look-back period from the first date of fill for the LM. Claims were stratified into age cohorts of "under 16 years" and " 16 years and older." Emergency room (ER) claims for patients utilizing LMs, ICSs, and patients on both LM and ICS were retrieved for analysis from the medical claims database for the same 12-month study period.
\end{abstract}

RESULTS: More than $89 \%$ of new LM starts had no history of an ICS in the claims database. Overall, $61 \%$ of all (new and existing) LM patients did not have a claim for an ICS in their drug claims profile during the study period. An estimated $25 \%$ of LM utilization was not for asthma. No differences in ER utilization were found between ICS users and LM users; however, the ER utilization rate (0.090 ER visits per patient per year) was lower with combination therapy compared with monotherapy with ICS $(0.110$ ER visits per patient per year, $P=0.001)$ or $L M$ $(0.119$ emergency room visits per patient per year, $P<0.001)$.

CONCLUSIONS: The majority of LM use in this health plan was initial monotherapy, contrary to national treatment guidelines for asthma. At the time of the study, the apparent off-label use of LM for allergic rhinitis was significant for this health plan.

KEYWORDS: Leukotriene modifiers, Inhaled steroids, Beta-agonists, Low-sedating antihistamines, Emergency room utilization

J Manag Care Pharm. 2004;10(2):115-21
$\mathrm{L}$

eukotriene modifiers (LM) include the 2 leukotriene receptor antagonists—zafirlukast (Accolate, approved by the FDA in September 1996) and montelukast sodium (Singulair, approved in February 1998) —and the leukotriene formation inhibitor zileuton (Zyflo, approved in January 1997). ${ }^{1}$ The LM class of drugs was a new addition to the pharmacotherapy armamentarium in the treatment of asthma. Since 1996, the utilization of LMs has grown because of their ease of administration (including once-daily dosing) and some largely unsubstantiated concern among physicians and patients over the perceived adverse effects from the long-term use of corticosteroids. Numerous clinical studies have also shown that LMs, when compared with inhaled corticosteroids (ICSs) for the treatment of asthma, are associated with higher hospital event rates and asthma care costs and increased use of shortacting beta-agonists. ${ }^{2-4}$

There has been some supposition that the oral formulation of LMs will be associated with better compliance compared with the inhaled formulation of ICS agents. ${ }^{5}$ While there is some support for this in the literature, it has yet to be shown that greater adherence results in superior clinical outcomes. ${ }^{6,7}$ Sherman et al. found that, while compliance was better for LMs, it was suboptimal for both LMs and ICSs. ${ }^{7}$ Bukstein found, in a small study, statistically significant better compliance with LMs as compared with ICSs in mild asthmatic children; however, there was no difference in any outcome measure between the ICSs and LMs. ${ }^{8}$ More work is required to determine the extent to which clinical outcomes might be improved with increased LM compliance.

Better treatment outcomes with ICSs and no current evidence of compliance benefits associated with LMs supports the use of ICSs as the drugs of choice in the treatment of asthma. ${ }^{9,10}$ The National Asthma Education and Prevention Program Expert Panel Report (NAEPP EPR), sponsored by the National

\section{Authors}

PAUL G. LAKOMSKI, RPh, MBA, is director, drug utilization review programs, and MONA CHITRE, PharmD, CGP, is director, clinical programs, for FLRx, the pharmacy management division of Excellus Health Plan, Inc., Rochester, New York.

AUTHOR CORRESPONDENCE: Paul G. Lakomski, RPh MBA, Director, Drug Utilization Review Programs, Excellus Health Plan, Inc., 344 South Warren St., Syracuse, NY 13202. Tel: (315) 671-7098; Fax: (315) 671-7095;

E-mail: paul.lakomski@flrx.com

Copyright $($ 2004, Academy of Managed Care Pharmacy. All rights reserved. 
(TABLE 1$)$ NAEPP-EPR2 Goals of Asthma Therapy ${ }^{\star}$

- Prevent chronic and troublesome symptoms (e.g., coughing or

breathlessness in the night, in the early morning, or after exertion)

- Maintain (near) "normal" pulmonary function

- Maintain normal activity levels (including exercise and other physical activity)

- Prevent recurrent exacerbations of asthma and minimize the need for emergency department visits or hospitalizations.

- Provide optimal pharmacotherapy with minimal or no adverse effects

- Meet patients' and families' expectations of and satisfaction with asthma care

* National Asthma Education and Prevention Program (NAEPP) Expert Report sponsored by the National Heart, Lung, and Blood Institute (NHLBI). NAEPP EPR (Expert Panel Report)-2. The complete guidelines are available at:

www.nhlbi.nih.gov/guidelines/asthma/index.htm.z.

Heart, Lung, and Blood Institute on the diagnosis and management of asthma, outlines 6 general goals of asthma therapy (Table 1) and provides a therapeutic algorithm designed to achieve these goals. The NAEPP EPR 2 in 1997 was issued shortly after the commercial availability of the first LM, zafirlukast. In 2002, an update to the NAEPP EPR 2 was released on selected topics. LMs were addressed in the update: "The LTRAs have been demonstrated to provide statistically significant but modest improvements in lung function when used as monotherapy in both adults and children. ... When comparing overall efficacy of LTRAs to inhaled corticosteroids in adult patients with persistent asthma, most outcome measures significantly and clearly favored inhaled corticosteroids. ..." The NAEPP EPR 2 was also modified to recommend LMs as an alternative but not preferred treatment to ICS when "... patient circumstances regarding administration of inhaled corticosteroids warrants selection of oral treatment"10 (see Figure 1, abstracted from http://www.nhlbi.nih.gov/guidelines/asthma/ execsumm.pdf).

First approved in February 1998 for use in asthma, on December 31, 2002, montelukast sodium became the first LM to be approved by the U.S. Food and Drug Administration (FDA) for use in allergic rhinitis. The coincident movement of the leading low-sedating antihistamines ([LSA] loratadine, Claritin) to over-the-counter (OTC) status, beginning in the same month (December 2002), precipitated concern in our health plan about the potential for increased utilization of LMs as a prescription alternative to OTC loratadine. Currently, the role of LMs in the treatment of allergic rhinitis remains inconclusive. The new allergic rhinitis indication for montelukast was not approved for concomitant use with antihistamines.

Available clinical guidelines ${ }^{11-13}$ for allergic rhinitis support the use of intranasal steroids as preferred therapy for moderate to severe allergic rhinitis. In addition, it has been shown that, in the treatment of seasonal allergic rhinitis, the clinical effects of the combination of an LM with an oral antihistamine are not significantly different from the use of an oral antihistamine alone. ${ }^{14}$ Studies have also shown that, in patients with seasonal allergic rhinitis, intranasal steroids are more effective than an LM-antihistamine combination for the reduction of pollen-induced nasal eosinophilic inflammation and for control of nasal symptoms. ${ }^{15}$

\section{Objective}

LMs were in the top 20 drug classes for cost during 2002 for our health plan and in the top 35 for prescription count; health plan costs had increased more than 13\% in 2002 compared with the previous year. Due to the rise in utilization and costs and reports in the literature of possible emergency room (ER) visits precipitated by the drug therapy change from ICSs to LMs ${ }^{16}$ our Drug Utilization Review (DUR) committee requested a review to assess the current utilization of LMs. The objective was to assess the utilization of LMs in the context of the current recommended guidelines for asthma and allergic rhinitis and to consider possible changes in formulary or tier copayment placement. A subanalysis was undertaken to assess possible differences in ER rates for those patients on ICS, LM, or both.

\section{Methods}

The prescription claims database for a 444,376-patient managed care organization in Upstate New York was used to examine utilization data for patients who received prescriptions for LMs zafirlukast, montelukast, and zileuton. Both new LM starts (defined as no claim in history 180 days prior to index date) and continued LM utilization between the dates of September 1, 2001, and August 31, 2002, were collected. The date of the first claim in history during the specified time frame served as the index date. Corresponding ER utilization was determined by the existence of medical claims with current procedural terminology (CPT) codes 99281 through 99285 (ER services for a new or established patient) for the same time frame (Figure 2).

Drug utilization was also grouped into 2 subsets: under age 16 years and age 16 years and older. New starts were further differentiated by the existence of an ICS (beclomethasone, budesonide, flunisolide, fluticasone, or triamcinolone) claim within 180 days prior to the start of the LM and those with an LSA (cetirizine, desloratadine, fexofenadine, or loratadine) in the claim history 180 days prior to the start of the LM. Total LM utilization was also differentiated by existence of an ICS, LSA, or beta-agonist (albuterol, bitolterol, formoterol, isoetharine, levalbuterol, metaproterenol, pirbuterol, salmeterol, or terbutaline) claim in the profile between September 1, 2001, and August 31, 2002. Rates and proportions were assessed for statistical significance using a test of homogeneity of proportions (chi-square goodness-of-fit test). Because of the large numbers, the data were assumed to be normally distributed.

\section{Results}

\section{New Starts: Leukotriene Modifiers,} Inhaled Steroids and Low-Sedating Antihistamine

In the population studied, $89.4 \%$ of new starts on LMs did not 
Classify Severity: Clinical Features Before Treatment or Adequate Control
Medications Required To Maintain

Long-Term Control

Daily Medications

- Preferred treatment:

High-dose inhaled corticosteroids

AND

Long-acting inhaled beta 2 -agonists

AND, if needed.

- Corticosteroid tablets or syrup long term $(2 \mathrm{mg} / \mathrm{kg} /$ day, generally do not exceed $60 \mathrm{mg}$ per day). (Make repeat attempts to reduce systemic corticosteroids and maintain control with high-dose inhaled corticosteroids.)

- Preferred treatment:

Low-to-medium dose inhaled corticosteroids and longacting inhaled beta 2 -agonists.

n Alternative treatment (listed alphabetically):

- Increase inhaled corticosteroids within medium-dose range OR

- Low-to-medium dose inhaled corticosteroids and either leukotriene modifier or theophylline.

If needed (particularly in patients with recurring severe exacerbations)

- Preferred treatment:

Increase inhaled corticosteroids within medium-dose range and add long-acting inhaled beta ${ }_{2}$-agonists.

- Alternative treatment (listed alphabetically):

- Increase inhaled corticosteroids within medium-dose range and add either leukotriene modifier or theophylline.

- Preferred treatment: Low-dose inhaled corticosteroids.

- Alternative treatment (listed alphabetically): cromolyn, leukotriene modifier, nedocromil, OR sustained-release theophylline to serum concentration of $5-15 \mathrm{mcg} / \mathrm{ml}$.

- No daily medication needed.

- Severe exacerbations may occur, separated by long periods of normal lung function and no symptoms. A course of systemic corticosteroids is recommended,

Mild Intermittent

$\frac{\leq 2 \text { days } / \text { week }}{\leq 2 \text { nights } / \text { month }} \quad \frac{\geq 80 \%}{<20 \%}$

Quick Relief

- Short-acting bronchodilator: 2-4 puffs short-acting inhaled beta 2 -agonists as needed for symptoms.

Quick Relief

- Intensity of treatment will depend on severity of exacerbation; up to 3 treatments at 20-minute intervals or a single nebulizer treatment as needed. Course of systemic corticosteroids may be needed.

- Use of short-acting beta -agonists $>2$ times a week in intermittent asthma (daily, or increasing use in persistent asthma) may indicate the need to initiate (increase) long-term-control therapy.

Step down

Review treatment every 1 to 6 months; a gradual stepwise reduction in treatment may be possible.

Step up

If control is not maintained, consider step up. First, review patient medication technique, adherence, and environmental control.

\section{Goals of Therapy: Asthma Control}

- Minimal or no chronic symptoms day or night

- Minimal or no exacerbations

- No limitations on activities; no school/work missed

Maintain (near) normal pulmonary function

Minimal use of short-acting inhaled beta-agonist

- Minimal or no adverse effects from medications
Note

- The stepwise approach is meant to assist, not replace, the clinical decisionmaking required to meet individual patient needs.

- Classify severity: assign patient to most severe step in which any feature occurs (PEF is \% of personal best: FEV 1 is \% predicted).

- Gain control as quickly as possible (consider a short course of systemic corticosteroids); then step down to the least medication necessary to maintain control.

- Minimize use of short-acting inhaled beta ${ }_{2}$-agonists. Overreliance on short-acting inhaled beta 2 -agonists (e.g., use of approximately one canister a month even if not using it every day indicates inadequate control of asthma and the need to initiate or intensify long-term-control therapy

- Provide education on self-management and controlling environımental factors that make asthma worse (e.g., allergens and irritants).

Refer to an asthma specialist if there are difficulties controlling asthma or if step 4 care is required. Referral may be considered if step 3 care is required. 
TABLE 2 New Start Patients* on Leukotriene Modifiers (LMs)

\begin{tabular}{|c|c|c|c|}
\hline & & $\begin{array}{l}\text { No ICS } \dagger \\
\text { (180 Days Prior } \\
\text { to Index Date) }\end{array}$ & $\begin{array}{l}\text { With LSA } \\
(180 \text { Days } \\
\text { Prior to } \\
\text { Index Date) }\end{array}$ \\
\hline Age Group & $\begin{array}{c}\text { No. of } \\
\text { Patients }\end{array}$ & $\begin{array}{c}\text { No. of } \\
\text { Patients (\%) }\end{array}$ & $\begin{array}{c}\text { No. of } \\
\text { Patients (\%) }\end{array}$ \\
\hline 16 and over & 1,174 & $1,081(92.1)$ & $258(22.0)$ \\
\hline Under 16 & 908 & $781(86.0) \S$ & $266(29.3)$ \\
\hline Total & 2,082 & 1,862 (89.4) & $524(25.2)$ \\
\hline \multicolumn{4}{|c|}{$\begin{array}{l}\text { * New starts were defined as no LM claim in the preceding } 180 \text { days. } \\
\text { † ICS }=\text { inhaled corticosteroid. } \\
\text { †LSA }=\text { low-sedating antihistamine. } \\
\S P<0.001 \text { for the comparison between the } 2 \text { age groups. }\end{array}$} \\
\hline
\end{tabular}

\section{TABLE 3 Total Leukotriene Modifier Utilization}

\begin{tabular}{|c|c|c|c|c|}
\hline Drug & $\begin{array}{c}\text { Patient Count } \\
\text { Age } 16 \\
\text { and Older }\end{array}$ & $\begin{array}{c}\% \text { of } \\
\text { Total }^{*}\end{array}$ & $\begin{array}{l}\text { Patient } \\
\text { Count Under } \\
\text { Age } 16\end{array}$ & $\begin{array}{c}\% \text { of } \\
\text { Total }^{*}\end{array}$ \\
\hline $\begin{array}{l}\text { Zileuton } \\
\quad 600 \mathrm{mg} \mathrm{tab}\end{array}$ & 38 & 0.76 & 0 & 0.00 \\
\hline $\begin{array}{l}\text { Montelukast } \\
10 \mathrm{mg} \text { tab } \\
4 \mathrm{mg} \text { chew tab } \\
5 \mathrm{mg} \text { chew tab }\end{array}$ & $\begin{array}{r}4,205 \\
2 \\
47\end{array}$ & $\begin{array}{r}83.68 \\
0.04 \\
0.94\end{array}$ & $\begin{array}{r}732 \\
804 \\
1,916\end{array}$ & $\begin{array}{r}21.05 \\
23.12 \\
55.1\end{array}$ \\
\hline $\begin{array}{l}\text { Zafirlukast } \\
\qquad 10 \mathrm{mg} \mathrm{tab} \\
20 \mathrm{mg} \text { tab }\end{array}$ & $\begin{array}{r}18 \\
715\end{array}$ & $\begin{array}{r}0.36 \\
14.23\end{array}$ & $\begin{array}{l}10 \\
15\end{array}$ & $\begin{array}{l}0.29 \\
0.43\end{array}$ \\
\hline Total $\dagger$ & 5,025 & & 3,477 & \\
\hline
\end{tabular}

* Totals may not equal $100 \%$ due to rounding.

+ A patient could be counted twice, or more, if, during the observation

period, the patient had claims for different agents (i.e., drug switches).

\section{TABLE 4 Utilization of Inhaled Steroids, Low-Sedating Antihistamines and Beta-Agonists in Patients Receiving Leukotriene Modifiers}

\begin{tabular}{|c|c|c|c|c|}
\hline Age Group & $\begin{array}{c}\text { No. of } \\
\text { Patients }\end{array}$ & $\begin{array}{l}\text { No Steroid } \\
\text { Inhaler } \\
\text { Claim in } \\
\text { History (\%) } \\
\text { No. of } \\
\text { Patients }\end{array}$ & $\begin{array}{c}\text { With Low- } \\
\text { Sedating } \\
\text { Antihistamine } \\
\text { Claim } \\
\text { History (\%) } \\
\text { No. of } \\
\text { Patients }\end{array}$ & $\begin{array}{c}\text { Beta- } \\
\text { Agonist } \\
\text { Claim in } \\
\text { History (\%) } \\
\text { No. of } \\
\text { Patients }\end{array}$ \\
\hline 16 and older & 4,968 & $3,105(62.5)$ & $2,045(41.2)$ & $3,718(74.8)$ \\
\hline Under 16 & 3,324 & $2,005(60.3)$ & $1,680(50.5)$ & $2,548(76.7)$ \\
\hline Total & 8,292 & $5,110(61.6)$ & $3,725(44.9)$ & $6,266(75.6)$ \\
\hline
\end{tabular}

have an ICS claim in their profile 180 days prior to the index date (Table 2). The percentage was higher in the age-16-yearsand-older group (92.1\%) than was found in the under-age-16years cohort $(86.0 \%, P<0.001)$. This appeared to be contrary to anecdotal information received from committee physicians, where there was some concern regarding long-term steroid use in pediatric patients. Direct-to-consumer (DTC) advertising could play a role in greater use of LM in the age-16-years-andolder population, with adults possibly seeking the medication in response to an advertisement. Juvenile and pediatric populations are generally not the focus for this type of consumer advertising, though we have no data to corroborate this supposition of the effect of DTC advertising on LM utilization. These findings suggest that current prescribing practice for our health plan patients involves the use of LMs as initial maintenance therapy and not as add-on therapy to inhaled steroids.

LMs are not indicated for concomitant use with LSAs, and research has shown no additional benefit when combined with LSAs. Overall, 25.2\% of new LM starts had existing or prior use of LSAs (defined as having a claim in history 180 days prior to index date). In the under-age-16-years group, there was a greater percentage of patients with existing LSA claims in history $(29.3 \%$ versus $22.0 \%, P<0.001)$. This finding was not unexpected. The occurrence of allergic rhinitis with asthma has been estimated at anywhere from $6.2 \%$ to $95 \%$ and has been shown to be an independent risk factor for asthma in adults. ${ }^{17}$ With the FDA approval of montelukast for allergic rhinitis, the potential for simplifying therapy by treating 2 different disease states with 1 medication may exist; however, the modest clinical benefit that LMs appear to have in both asthma and allergic rhinitis may limit this possibility. (Table 3 shows the distribution of LM utilization by drug and dose.)

For the total population of users of LMs, $61.6 \%$ did not have an inhaled steroid claim in their history during the 12-month study period. This would indicate that monotherapy with LMs is common. We did not investigate if an LM was being used concomitantly with long-acting beta-agonists.

The entire population of users of LM agents (both new starts and existing utilizers) were examined to identify the concomitant use of an ICS, LSA, or beta-agonist. Overall, $75.6 \%$ of patients with a claim for an LM did have a prescription filled for a long- or short-acting beta-agonist within the 12-month time frame of the observation period (Table 4). The existence of a beta-agonist claim for a patient was added as a proxy for a diagnosis of asthma. Standard of care for asthmatics includes the availability of an acute relief medication at all times. It was recognized that this is not a specific method of identifying the intended use for the medication. Mild-controlled asthmatics may not have the need for a beta-agonist prescription filled during a 12-month time frame. Also, beta-agonists are commonly used for acute, episodic care of upper respiratory infections and cough in nonasthmatic patients. While acknowledging the 


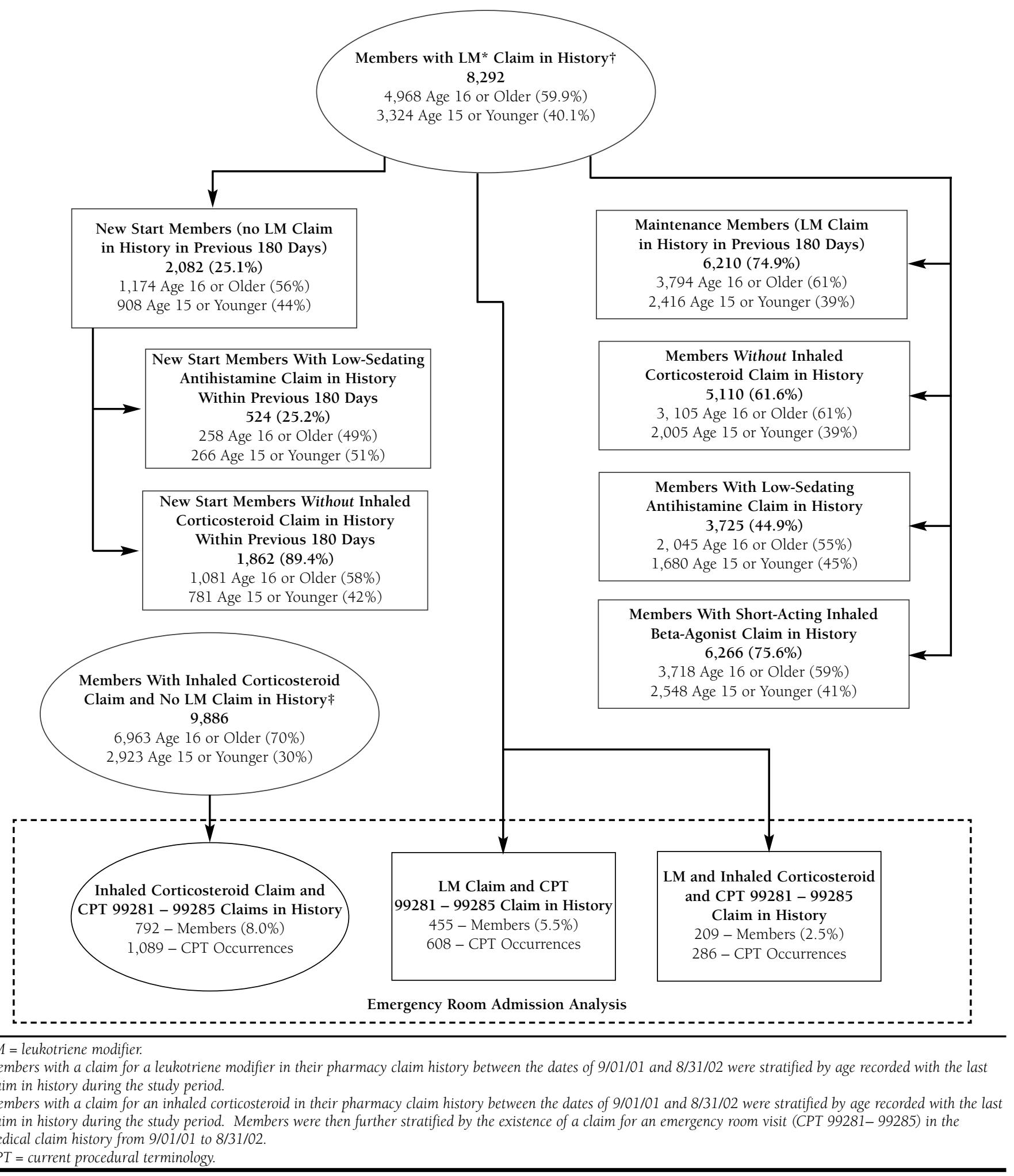




\section{TABLE 5 Emergency Room (ER) Visits in Users of Leukotriene Modifiers (LMs) and/or Inhaled Corticosteroids (ICSS)}

\begin{tabular}{|c|c|c|c|c|c|}
\hline & $\begin{array}{l}\text { No. ER } \\
\text { Visits }\end{array}$ & $\begin{array}{l}\text { No. ER } \\
\text { Patients }\end{array}$ & $\begin{array}{l}\text { No. ER Visits/ } \\
\text { ER Patient }\end{array}$ & $\begin{array}{l}\text { Total No. } \\
\text { Patients }\end{array}$ & $\begin{array}{r}\text { No. ER } \\
\text { Visits/ } \\
\text { Patient }\end{array}$ \\
\hline ICS monotherapy & 1,089 & 793 & 1.37 & 9,886 & 0.110 \\
\hline LM monotherapy & 608 & 455 & 1.34 & 5,110 & 0.119 \\
\hline ICS-LM dual therapy & 286 & 209 & 1.37 & 3,182 & $0.090 *$ \\
\hline \multicolumn{6}{|c|}{$\begin{array}{l}\text { Data from claims dated 9/01/01 to 8/31/02, rates per } 12 \text { months. } \\
\text { * Statistically significant compared with either ICS }(P=0.001) \text { or LM }(P<0.001) \\
\text { monotherapy. }\end{array}$} \\
\hline
\end{tabular}

inherent flaws in this measure, it is estimated that as much as $25 \%$ of current LM use could be for off-label utilization, i.e., allergic rhinitis.

This finding was used to support the implementation of a management strategy in which LM prescriptions required a tier-3 copayment unless the patient with the new LM claim had a prescription claim in history for an asthma medication. LM prescriptions were assigned a tier-2 copayment if the patient had received an asthma medication (short- or long-acting betaagonist, ICS, or theophylline) within 6 months prior to the LM claim. Otherwise, the LM prescription claims would adjudicate at the higher, tier-3 copayment. This management strategy for LMs was endorsed by the DUR committee and was consistent with the placement of all prescription LSAs in the third (highest) copayment tier by the health plan. While this is not an exact mechanism of determining intended use for the LM agents, it was felt to be a reasonable administrative strategy and one that did not impose a burden on internal plan resources, participating physicians, or their staff.

Due to published findings of increased ER use and hospitalizations for asthma when switching from an ICS to an LM, overall ER use by drug class was evaluated. ${ }^{16}$ Patients were stratified into ICS monotherapy, LM monotherapy, or concomitant ICS-LM therapy. The ER visits were not specifically limited to an asthma diagnosis, and no adjustments for case or asthma severity were made.

There was no difference in occurrences of ER claims with monotherapy with LM or ICS $(P=0.106)$ (Table 5$)$. Visits per ER patient and visits per patient were statistically similar among users of LM monotherapy and users of ICS monotherapy. ER utilization was lower (0.090 ER visits per patient per year) for combination therapy compared with either ICS monotherapy $(P=0.001)$ or LM monotherapy $(\mathrm{P}<0.001)$.

Since these patients were not stratified by disease severity, we cannot speculate about the relative therapeutic value of LM versus ICS as measured by the incidence of ER visits. Differences in average case severity could influence the finding of apparent lower ER utilization by users of combination LM and ICS. If LMs are being used as initial monotherapy, it could be argued that this group is more representative of mildto-moderate asthmatics while the ICS users may be more representative of moderate-to-severe asthmatics. If there was significant off-label utilization of LMs for allergic rhinitis as we have supposed, this could also affect the findings, causing the utilization rate for LMs to appear to be artificially low since ER admissions due to allergic rhinitis occur rarely. The possible impact of this variable was not quantified in our study.

The statistically significant lower ER utilization rate for those patients taking combination LM and ICS is worth further exploration. It was assumed that this cohort would include severe asthmatics and therefore would have higher ER utilization; conversely, dual therapy could represent more aggressively treated patients and be the reason for the lower utilization rate and possible better outcome. Since we did not measure case severity, we cannot determine if this is true. This finding supports the need for further research into combination therapy with ICS and LM.

\section{Limitations}

Our investigation examined only utilization of 3 LM agents, ICSs, and LSAs. We did not investigate if an LM was being used concomitantly with long-acting beta-agonists. The long-acting beta-agonist controller medications could influence either the LM or ICS groups if use patterns differed between the groups. Second, the existence of a short-acting beta-agonist claim is an imperfect determinant of intended LM use for asthma, since, for example, beta-agonists are commonly prescribed for upper respiratory infections; this proxy method could therefore result in an overestimate of the use of LMs for asthma. However, this tendency to overestimate the use of LMs for asthma may be offset by the fact that not all asthmatics will obtain a refill prescription of their acute relief (short-acting beta-agonist) medication in a 12-month study period.

We also could not verify concurrent use of an LM and an LSA; we determined only that their use occurred in the same patient within a 180-day period. This may not be reflective of true concomitant use and, alternatively, could represent therapy failures when the patients switch from one drug to another.

The incidence of ER visits reported in our study may overstate the rate of ER visits attributable to asthma since we did not assess the diagnostic codes on the ER claims. However, unless there was an underlying, disproportionate amount of concomitant disease in one group versus another, any difference in ER utilization could conceivably be attributable to the disease (asthma) or its treatment. Differences in medication utilization were found in the age-16-years-and-above cohort versus the below-age-16-years cohort. Since the ER utilization was not separated into these 2 age groups, we could not determine if age influenced ER utilization and might possibly confound the apparent differences in ER utilization associated with drug use. Disease severity was also not assessed in the ER utilization analysis. 


\section{Conclusions}

LMs in our population appear to be used commonly as initial therapy rather than as additional therapy to either reduce the dose of ICSs or improve asthma control. Utilization analysis showed possible significant use of LMs for indications other than asthma. Both findings are inconsistent with current clinical guidelines that recommend that ICS be used as first-line therapy (LM may be used as an alternative, add-on therapy). Our data did not show a significant difference in ER admission rates for ICS monotherapy versus LM monotherapy, but we did not adjust for disease severity. The ER utilization rate for patients on combination ICS and LM therapy was less than that for patients on therapy with either agent alone. The role of LMs and their place in therapy is evolving. Until the therapeutic role of LMs is better elucidated, these study findings will be used in our health plan to support intervention strategies and tier copayments to encourage the appropriate use of LMs, consistent with current evidence as manifest in clinical practice guidelines for both asthma and allergic rhinitis.

\section{ACKNOWLEDGMENTS}

Critical review of the manuscript and statistical assistance was provided by James Notaro, RPh, PhD, president, Clinical Support Services, Inc., West Seneca, New York, and Joel Owerbach, PharmD, vice president and chief pharmacy officer, FLRx, the pharmacy management division of Excellus Health Plan, Inc.

\section{DISCLOSURES}

No outside funding supported this research. Author Paul G. Lakomski served as principal author of the study. Study concept and design, analysis and interpretation of data, and drafting of the manuscript and its critical revision were the work of Lakomski and author Mona Chitre. Administrative, technical, and/or material support was provided by William Hayes, strategic data analyst, FLRx, the pharmacy management division of Excellus Health Plan, Inc.

\section{REFERENCES}

1. Drug Facts and Comparisons. CliniSphere version. St. Louis, MO: Facts and Comparisons; 2003.

2. Busse W, Raphael GD, Galant S, et al. Low dose fluticasone proprionate compared with montelukast for first line treatment of persistant asthma: a randomized clinical trial. J Allergy Clin Immunol. 2001;107(3):461-68.

3. Armstrong EP, Malone DC. Fluticasone is associated with lower asthmarelated costs than leukotriene modifiers in a real-world analysis.

Pharmacotherapy. 2002;22(9):1117-23.
4. Mamstrom K, Rodriguez-Gomez G, Guerra J, et al. Oral montelukast, inhaled beclomethasone, and placebo for chronic asthma. Ann Intern Med. 1999;130(6):487-95.

5. Strek ME, and the Antileukotriene Working Group. Consenus guidelines for asthma therapy. Ann Allergy Asthma Immunol. 2001;86(6)(suppl 1):40-44.

6. Williams B, Noonan G, Reiss TF, et al. Long-term asthma control with oral montelukast and inhaled beclomethasone for adults and children 6 years and older. Clin Exp Allergy. 2001;31(6):845-54.

7. Sherman J, Patel P, Hutson A, Chesrown S, Hendeles L. Adherence to oral montelukast and inhaled fluticasone in children with persistent asthma. Pharmacotherapy. 2001;21(12):1464-67.

8. Bukstein DA, Luskin AT, Bernstein A. "Real-world" effectiveness of daily controller medicine in children with mild persistent asthma. Ann Allergy Asthma Immunol. 2003;90(5):543-49.

9. National Asthma Education and Prevention Program Expert Panel Report 2: guidelines for the diagnosis and management of asthma. Available at: http://www.nhlbi.nih.gov/guidelines/asthma/asthgdln.htm. Accessed February 6, 2003

10. National Asthma Education and Prevention Program Expert Panel Report 2: guidelines for the diagnosis and management of asthma-update on selected topics; 2002. J Allergy Clin Immunol. 2002;110(5)(suppl):S141-S219.

11. Li JT, Pearlman DS, Nicklas RA, et al. Diagnosis and management of rhinitis: complete guidelines of the Joint Task Force on Practice Parameters in Allergy, Asthma, and Immunology. Ann Allergy Asthma Immunol. 1998;81 (5, pt 1):487-518.

12. Institute for Clinical Systems Improvement. Rhinitis. December 2001:33. Available at http://www.icsi.org/knowledge/detail.asp?catID=29+itemID=158 Accessed October 23, 2003.

13. Orlandi R, Baker J, Andreae M, et al. University of Michigan Health System. Allergic Rhinitis. Ann Arbor,MI: University of Michigan Health System; July 2002:12.

14. Nayak AS, Philip G, Lu S, Malice MP, Reiss TF, and the Montelukast Fall Rhinitis Investigator Group. Efficacy and tolerability of montelukast alone or in combination with loratadine in seasonal allergic rhinitis: a multicenter, randomized, double-blind, placebo controlled trial performed in the fall. Ann Allergy Asthma Immunol. 2002;88(6):592-600.

15. Pullerits T, Praks L, Ristioja V, Lotvall J. Comparison of a nasal glucocorticoid, antileukotriene, and a combination of antileukotriene and antihistamine in the treatment of seasonal allergic rhinitis. J Allergy Clin Immunol. 2002; 109(6):949-55.

16. Stempel DA, Pinto L, Stanford RH. The risk of hospitalization in patients with asthma switched from an inhaled corticosteroid to a leukotriene receptor antagonist. J Allergy Clin Immunol. 2002;110(1):39-41.

17. Togias A. Rhinitis and asthma: evidence for respiratory system integration. J Allergy Clin Immunol. 2003;111(6):1171-83; quiz 1184. 